\title{
Prevalence and cause of gestational diabetes mellitus in a tertiary care center in Kolar district: a population based study
}

\author{
Poojita Tummala ${ }^{1}$, Munikrishna M. ${ }^{1}$, Kiranmayee P. ${ }^{2 *}$
}

\begin{abstract}
${ }^{1}$ Department of Obstetrics and Gynecology, Sri Devaraj Urs Medical College, Kolar, Karnataka, India.
${ }^{2}$ Department of Cell Biology and Molecular Genetics, Sri Devaraj Urs Medical College, Kolar, Karnataka, India
\end{abstract}

Received: 24 April 2019

Accepted: 11 June 2019

\author{
*Correspondence: \\ Dr. Kiranmayee P., \\ E-mail: kiranmayee@sduu.ac.in
}

Copyright: (C) the author(s), publisher and licensee Medip Academy. This is an open-access article distributed under the terms of the Creative Commons Attribution Non-Commercial License, which permits unrestricted non-commercial use, distribution, and reproduction in any medium, provided the original work is properly cited.

\begin{abstract}
Background: Gestational diabetes mellitus (GDM) is carbohydrate intolerance at the onset of pregnancy which induces pathological short term or long term outcomes for both mother and baby. The aim of the present study was to know the prevalence of GDM in pregnant women who were attending the antenatal care (ANC) center at a tertiary care hospital in Kolar, Karnataka, India.

Methods: This prospective study was conducted in Department of Obstetrics and Gynecology, Sri Devaraj Urs Medical College, a constituent of Sri Devaraj Urs Academy of Higher Education and Research, Kolar, Karnataka, India. The duration of the study was two months. In this study, 108 pregnant women above 24 weeks of gestation were screened for GDM by oral glucose tolerance test. Fasting 2 milli liter blood was collected and were given 75 grams of glucose in 200 milli liters of water and asked to drink within 5 minutes. Again 2 milli liters venous blood was collected after 1 hour and 2 hours from all participants. Plasma sample was used for the estimation of glucose by glucose oxidase and peroxidase (GOD-POD) method.

Results: Out of 108,12 women $(11.1 \%)$ were diagnosed with GDM. The prevalence rate was higher in the age group of 26-30 years (41.6\%). Among 12 diabetic women, five (47.2\%) exercised regularly and seven (58.3\%) did not doing exercise. Out of 12 GDM subjects, eight of them had family history of diabetes in first degree relatives; among which one was hypertensive and five were suffering from thyroid problems.

Conclusions: In the present study, the prevalence of GDM was found to be $11.1 \%$. Prevalence of GDM might be influenced by increasing age, pre pregnancy weight, family history of diabetes, past history of pregnancy complications, status of literacy and exercise.
\end{abstract}

Keywords: Gestational diabetes mellitus, Glucose oxidase and peroxidase, Prevalence, Oral glucose tolerance test

\section{INTRODUCTION}

Gestational diabetes mellitus (GDM) is carbohydrate intolerance at the onset of pregnancy which induces pathological short term or long term outcomes for both mother and baby irrespective of the treatment with diet or insulin. ${ }^{1}$ GDM is multi factorial and can be influenced by obesity, family history, infertility treatment, polyhydramnios, recurrent urinary tract infection, history of still birth, prematurity, preeclampsia and genetic factors with certain polymorphisms notably TC7L2., Older maternal age, decrease in physical activity and adaptation of modern lifestyle in developing countries are also factors that can contribute to GDM. ${ }^{4,5}$ Pregnant women with GDM are at increased risk for future diabetes, pregnancy complications viz. abortion, pre-term 
labour and hydrammnios along with fetal complications viz. macrosomia, neural tube defects and cardiac anomalies. $^{6}$

Prevalence of GDM is expected to increase exponentially from 171 million in (2000) to 366 million (2030) globally. ${ }^{6}$ In India, recent data showed $16.55 \%$ of GDM prevalence. ${ }^{7}$ GDM prevalence is different in different ethnicities and it was found to be high in African, Indian and Hispanic women. ${ }^{8}$ Women who live in urban areas are more prone to GDM than those live in rural areas; obese women are more prone to have GDM globally. Women who have had GDM during first pregnancy are at high risk of developing GDM in their subsequent pregnancies and prevalence will be more in older women. ${ }^{9,10}$ Incidence of pre GDM and GDM may further complicate lead to complications viz. hypertension, renal diseases, thyroid dysfunction, eclampsia, fetal central nervous system (CNS) malformation, and fetal demise. $^{11,12}$

Screening of asymptomatic GDM should be done after 24 weeks of gestation as pathophysiology of the disease i.e. resistance to insulin will be seen from second trimester. The American Diabetes Association (ADA) recommends 2 step procedure for screening and diagnosis of GDM in selective population based on Carpenter and Coustan criteria for diagnosis of GDM; pregnant women with severe obesity, strong family history, previous GDM. Pregnant who fulfil criteria would be low risk and require no screening: less than 25 years, no family history of diabetes, normal pre-pregnancy weight and no history of poor obstetric outcome. Royal Australian and New Zealand College of obstetrics and gynecologists (RANZCOG) recommend universal screening for GDM. Screening of GDM is of 2 strategies; one step approach in which GDM is diagnosed by diagnostic Oral Glucose Tolerance Test (OGTT) without prior plasma or serum glucose screen. Second strategy is a two-step approach which is done by a diagnostic OGTT when first step is positive. , $^{73-16}$

During the last 4-8 gestational period, there may be chance of intrauterine fetal death due to higher levels of fasting glucose $(>105 \mathrm{mg} / \mathrm{dL}$ or $>5.8 \mathrm{mmol})$.GDM may associate with maternal hypertension and fetal growth disorders. The child born to a GDM woman is at increased jeopardy of obesity, diabetes in late adolescence and young adulthood and glucose intolerance.

Knowledge about GDM among mothers can help to decrease the birth complications and pathological outcomes. Findings from Carolan, Heather and Steele and Poth and Carolan showed that lack of appropriate knowledge about lifestyle and diet to prevent GDM contributes greatly to birth outcomes. Hence, the following study was taken up to know the prevalence and the associated risk factors for GDM in a tertiary care hospital.

\section{METHODS}

This prospective study was carried out at Department of Obstetrics and Gynecology, Sri Devaraj Urs Medical College, a constituent of Sri Devaraj Urs Academy of Higher Education, Kolar, Karnataka, India between July 2016 and September 2016. The work has been carried out on Indian Council of Medical Research (ICMR) Short Term Studentship (STS) programme. A total of 108 pregnant women who visiting the Department of Obstetrics and Gynecology for regular checkups were included in the study. Age of the study subjects was 1531 years. Pregnant women above 24 weeks of gestation i.e. in their 2nd and 3rd trimester were included in the study; pregnant women who are diagnosed previously with type 2 diabetes mellitus were excluded. The study was approved by institutional ethical committee (DMC/KLR/UDOME /IEC/127/2015-16). Blood samples for diagnosis of GDM were collected from 108 pregnant women above 24 weeks of gestation age after informed consent and history was documented in semi constructed proforma.

Under aseptic conditions, $2 \mathrm{ml}$ fasting blood samples were collected from 108 pregnant women from antecubital vein in resting condition into sodium fluoride tubes. All the participants were given $75 \mathrm{~g}$ of glucose in $200 \mathrm{~mL}$ of water and asked to drink during within 5 minutes intervals. Again venous blood was collected after 1 hour and 2 hour from all participants. Samples were centrifuges at 4000rpm for 10 minutes. Plasma sample was used for the estimation of glucose by glucose oxidase and peroxidase (GOD-POD) method in vitros FS 5.1 Dry chemistry autoanalyzer immediately.Diagnosis of GDM was done based on ADA criteria. Normal value for fasting glucose level is $>92 \mathrm{mg} / \mathrm{dL}$, for $1 \mathrm{sthr}$ sample is $180 \mathrm{mg} / \mathrm{dL}$ and for $2^{\text {nd }}$ hour is $>153 \mathrm{mg} / \mathrm{dL}$. Values obtained above the given range are considered abnormal. Any one abnormal value is considered as indicative of GDM.

\section{Statistical analysis}

The values were entered into excel form. Statistical analysis was performed by using institutional licensed version of IBM SPSS 20. This data includes comparison between GDM and the other risk factors. These factors were then statically analyzed by chi square test and the others by odds ratio.

\section{RESULTS}

In the present study, total number of pregnant women recruited was 108; baseline characteristics were shown in Table 1. Chi square and odds ratio ( $p$ values) of determinants were taken into consideration in the present study and were given in Table 2. GDM was diagnosed in 12 participants out of $108(11.1 \%)$ which was represented in Figure 1. The Chi square value was 98.11 and it was statistically significant $(\mathrm{p}=0.01)$. 
Table 1: Baseline characteristics of the participants.

\begin{tabular}{|ll|}
\hline Age & Results \\
\hline $15-20$ & $14(12.9 \%)$ \\
\hline $20-25$ & $51(47.2 \%)$ \\
\hline $26-30$ & $31(28.7 \%)$ \\
\hline $31-35$ & $21(19.4 \%)$ \\
\hline Physical activity & $66(61.1 \%)$ \\
\hline No & $42(38.8 \%)$ \\
\hline Yes & \\
\hline Family history of dm & $67(62.03 \%)$ \\
\hline No & $41(37.9 \%)$ \\
\hline Yes & \\
\hline Education & $5(4.62 \%)$ \\
\hline Illiterate & $3(2.77 \%)$ \\
\hline Primary education & $33(30.55 \%)$ \\
\hline Secondary education & $38(35.1 \%)$ \\
\hline PUC & $24(22.2 \%)$ \\
\hline Graduate & $5(4.62 \%)$ \\
\hline Post graduate & \\
\hline
\end{tabular}

Out of 108 participants; $47.2 \%$ women were between the age group of 20 to 25 years and majority of their education was $12^{\text {th }}$ standard. About $61.1 \%$ women were not doing any physical activity and $38.8 \%$ were doing regular physical activities. Around $75 \%$ of the participants were from rural background and most of their education level is low. Higher prevalence of GDM was seen in women with secondary education $(41 \%)$ and PUC (25\%). Only $25 \%$ illiterate women and $25 \%$ postgraduate women were GDM positive. The percentage of GDM in graduates was $9 \%$.

The highest number of participants was in the age group of 20- 25 years $(42.2 \%)$. The prevalence rate was high in the age group of $26-30$ years $(41.6 \%)$ followed by women in age group of $20-25$ years $(33.3 \%)$ compared to women aged between $15-20$ years and $31-35$ years $(25 \%)$. The Chi-square value is 5.44 and the $\mathrm{p}$ value was 0.14 . We also correlated GDM with age, but there was no significant relationship between GDM and age statistically.

Table 2: Chi square and Odds ratio ( $p$ values) of determinants of the present study.

\begin{tabular}{|c|c|c|c|c|c|c|}
\hline Determinants & Total (108) & GDM & & $\chi^{2}$ & $p$ value & Odds ratio \\
\hline Age (years) & & Yes & No & & & \\
\hline $15-20$ & 14 & 0 & 14 & \multirow{4}{*}{5.44} & \multirow{4}{*}{0.1423} & \\
\hline $20-25$ & 51 & 4 & 47 & & & \\
\hline $26-30$ & 31 & 5 & 26 & & & \\
\hline $31-35$ & 12 & 3 & 9 & & & \\
\hline \multicolumn{4}{|c|}{ Blood glucose level } & \multirow{3}{*}{98.11} & \multirow{3}{*}{0.001} & \\
\hline Normal & 96 & & & & & \\
\hline Positive & 12 & & & & & \\
\hline \multicolumn{4}{|c|}{ Physical activity } & & \multirow{3}{*}{0.8343} & \multirow{3}{*}{1.39} \\
\hline Yes & 66 & 7 & 59 & & & \\
\hline No & 42 & 5 & 37 & & & \\
\hline \multicolumn{4}{|l|}{ Family history } & & & \\
\hline Yes & 41 & 8 & 33 & & \multirow{2}{*}{0.0390} & \multirow{2}{*}{3.8182} \\
\hline No & 67 & 4 & 63 & & & \\
\hline
\end{tabular}

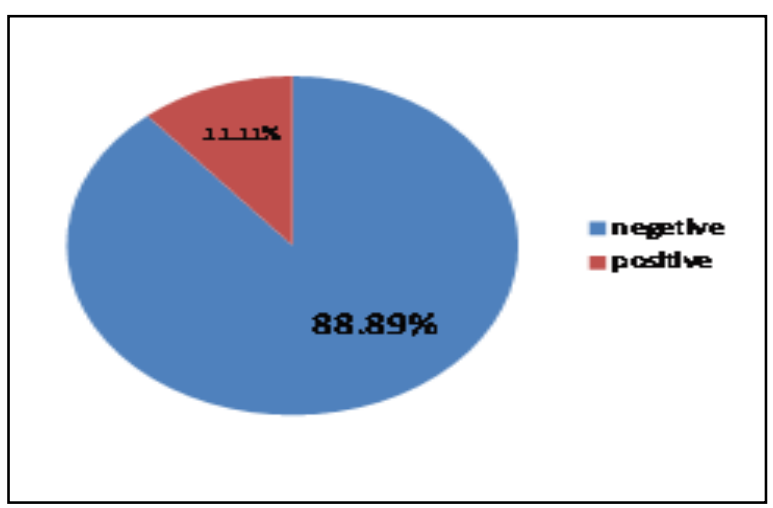

Figure 1: Frequency of diabetes in Kolar population.

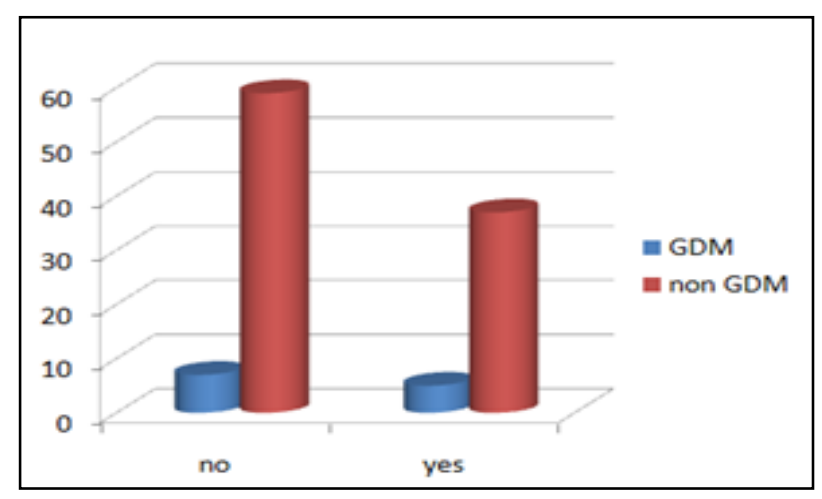

Figure 2: Relation between the physical activity and GDM. 
Among 12 GDM women, 5 (41.6\%) were doing regular exercise and $7(58.4 \%)$ were not doing exercise. In present study, it was documented that GDM was more prevalent in women who were not exercising (Figure 2).

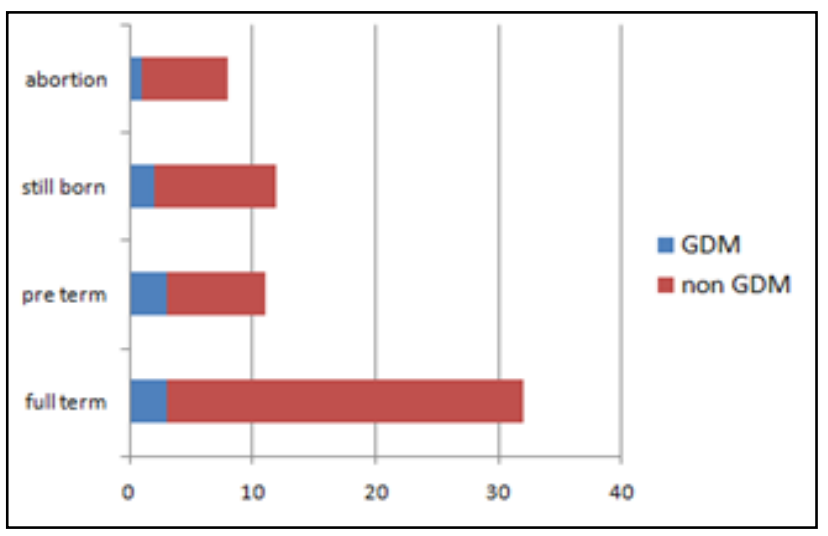

Figure 3: Relation between GDM and previous pregnancies.

Even though odds ratio (1.39) and p value (0.83) were not statically significant, GDM was prevalent in women who were not on exercise. In the present study, out of 12 diabetic women, three women with history of a preterm delivery, one woman with history of abortion and 2 women with history of still births were documented (Figure 3).

The participants were asked about the awareness of GDM and the consequences thereafter or its effects. Only 48 people out of 108 were aware of this (Figure 4).

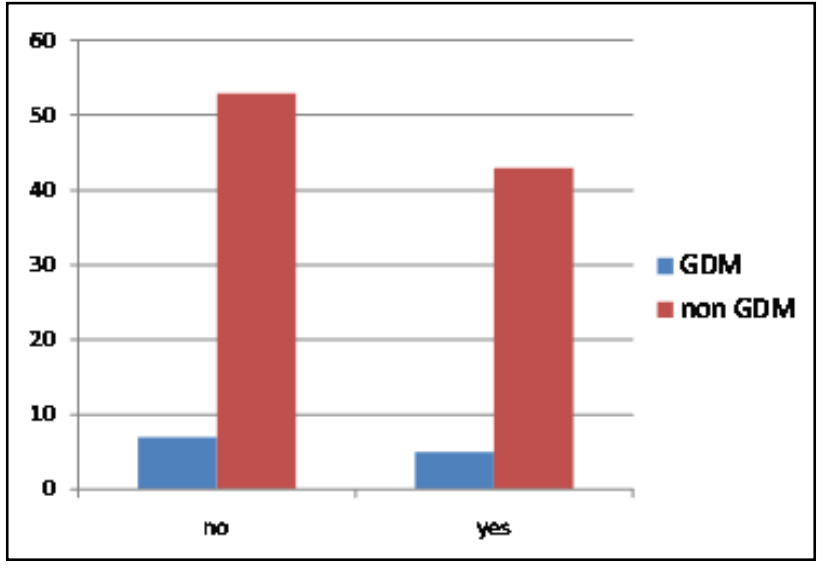

Figure 4: Relation between GDM and awareness of the participants about GDM.

Family history of the participants was taken into consideration. In the present study, out of 12,8 pregnant women had family history of diabetes in first degree relatives. The odds ratio was 3.81 and was statistically significant $(\mathrm{p}=0.03)$ (Figure 5). Out of 12 , only one was hypertensive and 5 were suffering from thyroid problems. No other significant problems were noted among the participants.

\section{DISCUSSION}

GDM in pregnant women is multi factorial can cause increased risk for future diabetes, pregnancy complications viz., abortion, pre-term labour and hydrammnios along with fetal complications viz., macrosomia, neural tube defects and cardiac anomalies.

In the present study, prevalence of GDM in Kolar was documented as $11.11 \%$; prevalence of GDM across the country was reported to be $16.5 \%$ with an increase from $2 \%$ to $7.6 \%$. $^{6}$ It is hypothecated that GDM cases will be increasing exponentially to 101.2 million by 2030 . In a random survey during 2002 to 2003, prevalence of GDM in major cities were reported as Aluva (21\%), Erode (18.8\%), Ludhiana (17.5\%), Chennai (16.2\%), Thiruvananthapuram (15\%) and Bengaluru (12\%). In India, out of 65 million diabetes and 77 million prediabetes, approximately $45 \%$ are women. It is estimated that about 4 million women are affected by GDM in India at any given point of time. ${ }^{17}$ Community based study in Tamilnaduduring 2008 reported that prevalence of GDM in urban population was $17.8 \%$, semi urban population was $13.8 \%$ and rural population was $9.9 \%$. The geographical differences in prevalence have been attributed due to differences in age and/or socioeconomic status of pregnant women in this regions. ${ }^{18}$

Maternal monitoring is one of the therapeutic strategies during pregnancy. Everyday blood glucose monitoring of self is the best choice or monitoring of postprandial glucose levels when women are under insulin treatment is superior to pre-prandial. In GDM, ketone monitoring in urine may be useful but not the urine glucose. Ultrasonography will assess the asymmetric fetal growth, in particular, in early third trimester that can benefit maternal insulin therapy.

In the present study, prevalence of GDM in women above 25 years was $66.6 \%$. In a study conducted in Kollam district showed prevalence of GDM in women above 25 years was $75 \%$. Thus there is a significant relationship between age and prevalence of GDM. Our study revealed that women with lesser extent of education are prone to get GDM and prevalence of GDM will be high in these pregnant women. Because of awareness about GDM and its consequences in educated women might be the reason for less prevalence of GDM.A study conducted by Rajput et al at Haryana during the year 2008, there was increased prevalence of GDM has been reported in educated women. ${ }^{19}$

In the present study, out of 66 pregnant women who were not doing regular exercise a part from household activities, 7 women were diagnosed for GDM with a prevalence rate of $10 \%$. Out of 42 pregnant women who were on regular exercise along with household activities 5 were diagnosed for GDM with a prevalence rate of $11.9 \%$. In a pilot study done to know the relationship between prevalence of GDM with regular exercise 
reported that $18 \%$ of pregnant women who were not on regular exercise were GDM positive. Even though there was no significant statistical relation between prevalence of GDM with regular exercise, the prevalence of GDM will be high in pregnant women who do not do regular exercise. $^{20}$

In the present study, prevalence of GDM was equal in pregnant women who had history of full term deliveries and pre term deliveries. The pregnant women who had absorption showed $80 \%$ prevalence for GDM. In a study conducted in south India during 2005 was reported that among 7 pregnant women who were diagnosed for GDM 6 pregnant women had history of miscarriage out of 980 participants. $^{21}$ The pregnant women with family history of diabetes in first degree relatives were 3.8 times prone to get GDM when compared to the women who do not have family history in this study. Diabetes can be transmitted through genetic factors from generations to generations might be the reason for the high prevalence of GDM in pregnant women with significant family history. Only 5.5\% women had other complications viz., thyroid problems and hypertension were diagnosed for GDM in the present study. Annual test for diabetes, individual exercise/physical activity, lifestyle modifications, looking after normal body weight are some of the long-term therapeutic concerns. If the patients develop symptoms of hyperglycemia, education of keeping optimal glycemic guideline, seeking medical attention are essential for the start of subsequent pregnancies.

\section{CONCLUSION}

The present study reported the prevalence of GDM was $11.1 \%$ in a tertiary care hospital, Kolar, Karnataka. Prevalence of GDM might be influenced by increasing age, high pre pregnancy weight, family history of diabetes, past history of maternal complications and other health complications and status of exercise. Large prospective studies recommended.

\section{ACKNOWLEDGMENTS}

Authors would like to thank ICMR (STS), SDUAHER management for their support, OBG department, and Central Diagnostic laboratory.

Funding: No funding sources Conflict of interest: None declared

Ethical approval: The study was approved by the Institutional Ethics Committee

\section{REFERENCES}

1. Dornhorst A, Rossi M. Risk and prevention of type 2 diabetes in women with gestational diabetes. Diabetes care. 1998;21(2):B43-9.

2. Bhat M, KNR, Sarma, SP, Menon S, CVS,SGK. Determinants of gestational diabetes mellitus: A case control study in a district tertiary care hospital in South India. Int $\mathbf{J}$ Diabetes Dev Ctries. 2010;30(2):91-6.

3. Zhang C, Bao W, Rong Y, Yang H, Bower K, Yeung $\mathrm{E}$, et al. Genetic variants andthe risk of gestational diabetes mellitus: A systematic review. Hum Reprod Update. 2013;19(4):376-90.

4. Martin JA, Hamilton BE, Sutton PD, Ventura SJ, Menacker F, Munson ML. Births: Final data for 2002. Natl Vital Stat Rep. 2003;52(10):1-113.

5. Pan XR, Yang WY, Li GW, Liu J. Prevalence of diabetes and its risk factors in China, 1994. National Diabetes prevention and Control Cooperative Group. Diabetes Care. 1997;20(11):1664-9.

6. Sreekanthan K, Belicita A, Rajendran K, Anil V. Prevalence of gestational diabetesmellitus in a medical college in south India: A pilot study. Indian J Clinical Practice. 2014;25(4); 342-7.

7. Seshiah V, Balaji V, Balaji MS, Sanjeevi CB, Green A. Gestational diabetes mellitus in India. J Assoc Physicians India. 2004;52:707-11.

8. Carolan M, Davey MA, Biro MA, Kealy M. Maternal age, ethnicity and gestational diabetes mellitus. Midwifery. 2012;28(6):778-3.

9. Stark Casagrande S, Rios Burrows N, Geiss LS, Brainbridge KE, Fradkin JE, Cowie CC. Diabetes knowledge and its relationship with achieving treatment recommendations in a national sample of people with type 2 diabetes. Diabetic Care. 2012;35(7):1556-65.

10. Chen Y, Quick WW, Yang W, Zhang Y, Baldwin A, Moran J, et al. Cost of gestational diabetes mellitus in the United States in 2007. Popul Health Manag. 2009;12(3):165-74.

11. Fong A, Serra A, Herrero T, Pan D, Ogunyemi D. Pre-gestational versus gestational diabetes: A population based study on clinical and demographic differences. J Diabetes Complications. 2014;28(1):29-34.

12. Xiang AH, Li BH, Black MH, Sacks DA, Buchanan TA, Jacobsen SJ, et al. Racial and ethnic disparities in diabetes risk after gestational diabetes mellitus. Diabetologia. 2011;54(12):3016-21.

13. Seshiah V, Balaji V, Madhuri S, Balaji MS, Aruna S, Sanjeevi CB, et al. One step procedure for screening and diagnosis of gestational diabetes mellitus. The $\mathbf{J}$ Obs Gyn India. 2005;55(6):525-9.

14. Nicholson WK, Fleisher LA, Fox HE, Powe NR. Screening for gestational diabetes mellitus: a decision and cost effectiveness analysis of four screening strategies. Diabetic Care. 2005;28:1482-4.

15. Meltzer SJ, Snyder J, Penrod JR, Nudi M, Morin L. Gestational diabetes mellitus screening and diagnosis: a prospective randomized controlled trail comparing costs of one-step and two-step methods. British J Obstet Gynec. 2010;117:407-15.

16. Carpenter MW, Coustan DR. Criteria for screening tests for gestational diabetes. Am J Obstet Gynecol. 1982;144:768-73. 
17. Standards of medical care in diabetes-2012. Diabetes care. 2012;35 (suppl 1).

18. Reddi RP, Jasmina B Screening and diagnosis of gestational diabetes mellitus, where do we stand. J Clinc Diagn Res. 2016;10(4):QE01-QE04.

19. Seshiah V, Balaji V, Balaji MS, Paneerselvam A, Arthi T, Thamizharasi M. Prevalence of gestational diabetes mellitus in South India (Tamil Nadu) - a community based study. J Assoc Physicians India. 2008;56:329-33.

20. Kayal A, Anjana RM, Mohan V. Gestational diabetes - An update from India. Diabetes Voice. 2013;58.
21. Rajput R, Yadav Y, Nanda S, Rajput M. Prevalence of gestational diabetes mellitus and associated risk factors at a tertiary care hospital in Haryana. The I J Medical Res. 2013;137(4):728-33.

Cite this article as: Tummala $\mathrm{P}$, Munikrishna $\mathrm{M}$, Kiranmayee P. Prevalence and cause of gestational diabetes mellitus in a tertiary care center in Kolar district: a population based study. Int J Reprod Contracept Obstet Gynecol 2019;8:2709-14. 